\title{
A case of isolated amyloid light-chain amyloidosis of the radial nerve
}

\author{
Ron Ron Cheng, MD, ${ }^{1}$ Ramin Eskandari, MD, ${ }^{1}$ Cynthia T. Welsh, MD, ${ }^{2}$ and Abhay K. Varma, MD ${ }^{1}$ \\ 'Department of Neurosciences, Division of Neurosurgery; and 2Department of Pathology and Laboratory Medicine, Medical \\ University of South Carolina, Charleston, South Carolina
}

Peripheral nerve involvement may be the first sign of systemic amyloid light-chain (AL) amyloidosis, a rare disease. Physical examination and electrodiagnostic testing are the mainstays of peripheral neuropathy evaluation at presentation. Sural nerve biopsy is performed in conjunction with serum and urine protein evaluation to differentiate between focal and systemic disease. Systemic disease is treated with a combination of chemotherapy, steroids, and stem cell transplantation. Isolated peripheral nerve disease is extremely rare.

The authors here report the case of an 80-year-old woman who presented with progressive right upper-extremity weakness due to right radial neuropathy discovered upon electrodiagnostic testing. Magnetic resonance neurography (MRN) revealed a focal lesion within the right radial nerve. She underwent radial nerve exploration and excision of an intraneural mass consisting of amyloid on histopathology, with mass spectrometry analysis diagnostic for AL amyloidosis. Noninvasive testing and clinical history did not suggest systemic involvement. This unique case of isolated peripheral nerve $\mathrm{AL}$ amyloidosis in the absence of signs and symptoms of systemic disease is described, and the literature demonstrating peripheral nerve involvement in AL amyloidosis is reviewed.

http://thejns.org/doi/abs/10.3171/2015.10.JNS151095

KEY WORDS peripheral nerve; amyloidosis; mononeuropathy; intraneural amyloidoma; magnetic resonance neurography

$A$ MYLOIDOSIS is a family of diseases characterized by abnormal extracellular deposition of protein fibrils in various organs and tissues. Amyloid light-chain (AL) amyloidosis is the most common form encountered and typically manifests as deposits in the kidneys, heart, liver, autonomic nervous structures, and/or peripheral nerves. Protein deposits cause organ damage by interrupting normal tissue architecture, and they also have direct cytotoxic cellular effects. ${ }^{12}$ The incidence is estimated to be approximately $5-12$ patients per million persons per year, and systemic disease generally requires chemotherapy. ${ }^{12}$

Peripheral neuropathy is seen in 15\%-20\% of systemic amyloidosis cases and can be the presenting symptom. ${ }^{1}$ Most cases of peripheral nerve AL amyloidosis reported in the literature are a component of systemic disease. . $^{1,3-8 \text {, }}$ ${ }_{10,11,13-16}$ We describe a unique case of isolated radial nerve $\mathrm{AL}$ amyloidosis presenting as an intraneural mass.

\section{Case Report}

History and Physical Examination

An 80-year-old right-handed woman presented with several years of weakness in her right arm. Within the 6 months prior to her neurosurgical evaluation, her initially mild difficulty in using her right hand progressed into marked weakness in that hand, resulting in an inability to play the piano. Additional symptoms included numbness in the dorsum of the right hand, as well as occasional nonradiating right-sided neck and shoulder pain. There was no history of trauma. A review of her systems was otherwise negative for constitutional symptoms such as weight loss, fevers, or fatigue. Physical examination revealed a right wrist drop, an inability to extend her fingers against gravity, wasting of the right brachioradialis muscle, and decreased sensation of the dorsal tissue between the first and second digits on the right.

ABBREVIATIONS $A L=$ amyloid light chain; $E M G=$ electromyography; $F L C=$ serum free light chain; $H D M=$ high-dose melphalan; IFE = immunofixation electrophoresis; $\mathrm{MRN}=$ magnetic resonance neurography; $\mathrm{SCT}=$ stem cell transplantation; SPEP = serum protein electrophoresis; $\mathrm{STIR}=$ short T1 inversion recovery.

SUBMITTED May 11, 2015. ACCEPTED October 5, 2015.

INCLUDE WHEN CITING Published online January 15, 2016; DOI: 10.3171/2015.10.JNS151095. 


\section{Diagnostic Testing}

A nerve conduction study was performed on bilateral upper extremities and demonstrated normal motor responses in the right median, right ulnar, and left radial nerves. There were normal sensory responses in the right median digit and right ulnar digit branches. Borderline reduced amplitude but normal distal latency was observed in the left radial nerve. There was absent sensory response, reduced motor amplitude, and conduction velocity slowing after proximal stimulation with normal distal latency in the right radial nerve. These findings were interpreted as a severe distal right radial sensorimotor mononeuropathy. Electromyography (EMG) revealed denervation of muscle groups supplied by the radial nerve distal to the elbow. Electromyographic testing of the right triceps, hand muscles, and biceps was normal. Magnetic resonance neurography (MRN) without contrast of the right upper extremity demonstrated focally increased signal within the radial nerve at the level of the lateral epicondyle on both T2-weighted and short T1 inversion recovery (STIR) sequences (Fig. 1), along with diffuse atrophy of the extensor carpi radialis longus, brachioradialis, and supinator muscles.

\section{Intervention, Operative Findings, and Pathological Diagnosis}

Exploration of the right arm both proximal and distal to the lateral epicondyle revealed a fibrous band compressing the radial nerve just as it exited the spiral groove of the humerus and pierced the intermuscular septum into the anterior compartment of the forearm. Distal to this fibrous band, the radial nerve appeared swollen. Internal neurolysis demonstrated a 5- to 6-mm intraneural mass bordered by stretched nerve fascicles (Fig. 2). The radial nerve was stimulated proximal and distal to this mass, with an EMG response in the brachioradialis muscle but without visible contraction. The mass was excised via intracapsular decompression and sent for pathological examination. Postexcision, repeat radial nerve stimulation proximal and distal to the lesion once again revealed an EMG response in the brachioradialis muscle without visible contraction.

Histopathology revealed amyloid deposits infiltrating both vessel walls and nerve (Fig. 3A). Congo red staining confirmed the presence of amyloid with apple-green birefringence on polarization (Fig. 3B and C). Subsequent mass spectrometry analysis of the specimen was consistent with AL $\kappa$ light chains.
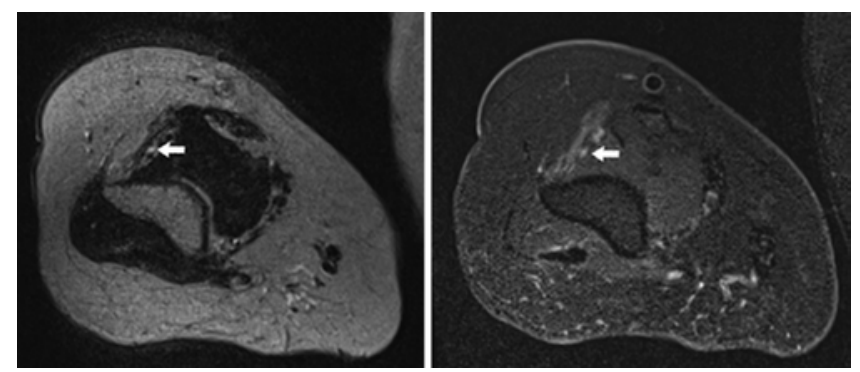

FIG. 1. Magnetic resonance neurography showing focus of increased signal (arrow) within the right radial nerve on both axial T2-weighted (left) and STIR (right) sequences.

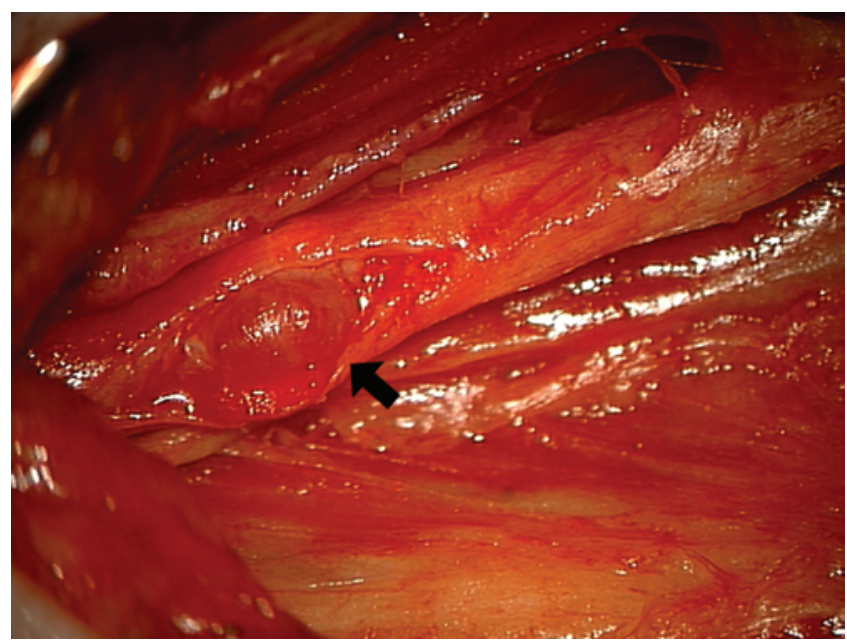

FIG. 2. Intraoperative photograph showing the radial nerve after dissection and an intraneural mass (arrow). Figure is available in color online only.

\section{Follow-Up}

Subsequent hematological evaluation demonstrated no evidence of systemic disease based on serum protein electrophoresis (SPEP), immunofixation electrophoresis (IFE), and serum free light chain (FLC) assay. The patient refused bone marrow biopsy for definitive diagnosis because she did not wish to undergo chemotherapy. Given the diagnostic information available, consensus opinion was that her disease was isolated to the radial nerve.

Postoperatively, the patient had an increased range of motion in her right third and fourth fingers as well as improved sensation in the right hand. Five months postoperatively, she demonstrated improvement in motor function through an ability to extend her index and fifth fingers against gravity.

\section{Literature Review}

A literature review was performed by searching the PubMed database with the terms "peripheral nerve AL amyloidosis" and filtering for results available in English. This yielded 40 search results, 13 of which reported discrete numbers of patients with AL amyloidosis involving the peripheral nervous system. Four results described cranial nerve amyloid deposits, which were not included in our review. These 13 references are briefly summarized in Table 1. In all but a few reports, the pattern of nerve involvement is specified.

Vucic et al. reported 4 cases with atypical presentations of neuropathy due to primary AL amyloidosis. ${ }^{15}$ Two patients in their series presented with a painful sensorimotor polyneuropathy, 1 with multiple mononeuropathies, and 1 with primary demyelinating polyradiculoneuropathy. After an extensive workup of their neuropathy, all patients were found to have monoclonal protein on serum IFE as well as tissue amyloid suggesting systemic AL amyloidosis. Of note, the third patient underwent thoracic MRI, which revealed thickening of the right brachial plexus. Diagnosis in all cases was achieved between 2 and 4 years 

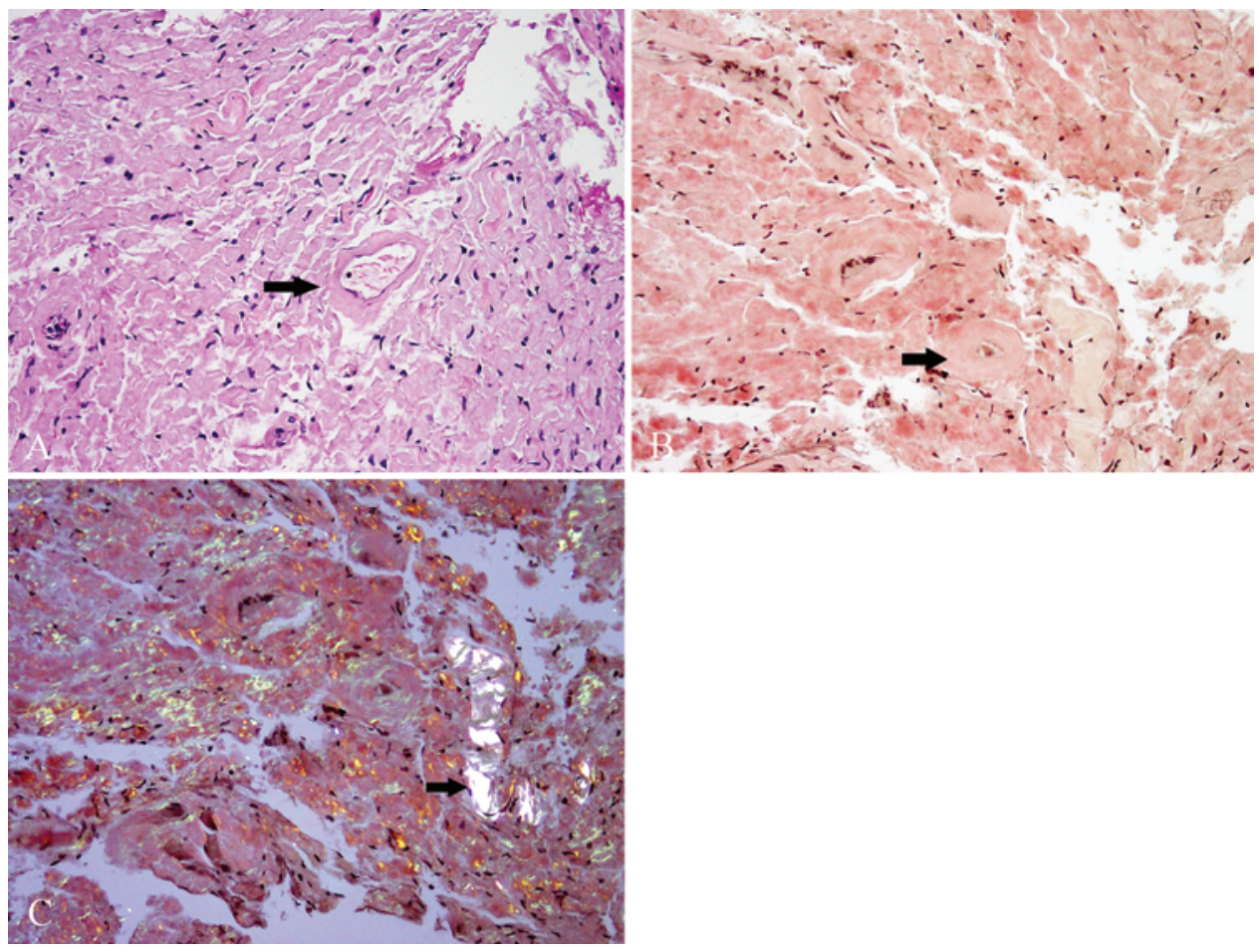

FIG. 3. Histopathological examination under light microscopy demonstrates amyloid deposits infiltrating a vessel wall (A, arrow) and nerve, amyloid deposit in a vessel wall (B, arrow) and diffusely infiltrating nerve, and apple-green birefringence in nerve and vessels, with sporadic collagen deposits $(\mathbf{C}$, arrow). $\mathrm{H} \& \mathrm{E}(\mathrm{A})$ and Congo red stain $(\mathrm{B}$ and $\mathrm{C}$ ), original magnification $\times 20$. Figure is available in color online only.

TABLE 1. Review of the literature reporting peripheral nerve $A L$ amyloidosis*

\begin{tabular}{|c|c|c|}
\hline Authors \& Year & $\begin{array}{c}\text { No. of Patients } \\
\text { w/ Peripheral } \\
\text { Nerve } \\
\text { Involvement }\end{array}$ & Pattern of Involvement \\
\hline Zanusso et al., 1992 & 3 & Polyneuropathy \\
\hline Conaghan et al., 2000 & 1 & Recurrent laryngeal nerve \\
\hline Dispenzieri et al., 2001 & 39 & Nerve involvement \\
\hline Berghoff et al., 2003 & 7 & Polyneuropathy \\
\hline Vucic et al., 2003 & 4 & $\begin{array}{l}\text { Polyneuropathy, multiple } \\
\text { mononeuropathies }\end{array}$ \\
\hline Dispenzieri et al., 2004 & 22 & Peripheral neuropathy \\
\hline Sarkar et al., 2005 & 1 & Peripheral neuropathy \\
\hline $\begin{array}{l}\text { Garces-Sanchez et al., } \\
2008\end{array}$ & 21 & Polyneuropathy \\
\hline Katoh et al., 2010 & 2 & Polyneuropathy \\
\hline Tracy et al., 2010 & 1 & Multiple mononeuropathies \\
\hline Matsuda et al., 2011 & 15 & $\begin{array}{l}\text { Polyneuropathy, bilateral } \\
\text { median nerves }\end{array}$ \\
\hline Luigetti et al., 2012 & 1 & Polyneuropathy \\
\hline Andrei et al., 2014 & 1 & Polyneuropathy \\
\hline
\end{tabular}

* No localized disease was reported in any of the studies. after symptom onset. These cases were reported because of peripheral neuropathy manifesting with neither cardiac nor renal symptoms of systemic AL amyloidosis. The first patient was treated with melphalan and prednisone, with stable symptoms and serum monoclonal protein concentration at 6 months. The second and third patients were treated with high-dose melphalan and stem cell transplantation with stable neurological deficits at 1 year and 6 months, respectively. The last patient died of respiratory failure prior to starting treatment. ${ }^{15}$

In comparison, the patient in our case had a similar delay in diagnosis of multiple years. She also lacked other common systemic manifestations of AL amyloidosis. Our case is unique because of the involvement of a single peripheral nerve and presentation as an intraneural mass. Besides electrodiagnostic testing, MRN was useful in demonstrating a focal nerve lesion consistent with clinical findings, as well as in guiding surgical intervention. Our patient did not demonstrate plasma cell dyscrasia and was not started on therapy postoperatively.

\section{Discussion}

Amyloid light-chain amyloidosis is the most common of the amyloidosis diagnoses. It is characterized by a higher ratio of $\lambda$-subtype/ $\kappa$-subtype light chain expression by plasma cells (nearly 3:1). ${ }^{12}$ Abnormal light chain expression leads to insoluble protein aggregates (amyloid) that deposit in organ tissue. The most commonly affected organs are the kidneys and heart. 
Diagnosis of systemic AL amyloidosis requires the presence of tissue amyloid and plasma cell dyscrasia. Tissue diagnosis can be made via a minimally invasive abdominal fat pad biopsy, with a reported sensitivity of $70 \%-85 \%$, or a sural nerve biopsy, with a sensitivity of 86\%-90\%. ${ }^{15}$ Plasma cell dyscrasia can be demonstrated by identifying monoclonal light chain via SPEP, urine protein electrophoresis (UPEP), IFE, or serum FLC assay. Bone marrow biopsy showing a predominance of plasma cells producing $\lambda$ or $\kappa$ light chains is also diagnostic. ${ }^{12}$ Traditionally, bone marrow biopsy has been necessary for a definitive diagnosis to evaluate plasma cell burden and exclude multiple myeloma. ${ }^{12}$ However, Katzmann et al. demonstrated in a study of 110 patients diagnosed with systemic AL amyloidosis that the combination of an abnormal FLC assay with a positive IFE was found in $99 \%$ of patients with a tissue-confirmed diagnosis of AL amyloidosis. ${ }^{9}$

Clinical manifestations of end-organ involvement include not only nephrotic syndrome and restrictive cardiomyopathy, but also hepatomegaly, orthostatic hypotension, and sensory neuropathy progressing to motor neuropathy. ${ }^{12}$ In a study of peripheral nerve involvement in primary systemic AL amyloidosis, Matsuda et al. found that polyneuropathy was generally symmetric and mostly sensory in nature; signs and symptoms of early disease were predominantly noted in the lower extremities, whereas later-stage polyneuropathy was characterized by autonomic dysfunction (for example, orthostatic hypotension and gastrointestinal distress). ${ }^{11}$

While physical examination (for example, extremity edema in renal disease and dyspnea on exertion in cardiomyopathy) and tissue biopsy are the most widely accepted methods to assess organ involvement in amyloidosis, multiple imaging modalities have been used to determine the presence of amyloid, particularly in the heart, including scintigraphy with technetium-99m pyrophosphate, echocardiography, and cardiac MRI. ${ }^{12}$ Cardiac MRI demonstrating delayed enhancement of myocardial interstitial space may help to distinguish between amyloid and hypertensive causes of ventricular hypertrophy, but MRI detection of amyloid in noncardiac tissues has unknown utility. ${ }^{12}$

The treatment of primary AL amyloidosis is primarily aimed at reducing the systemic amyloid protein burden by addressing the plasma cell dyscrasia; supportive therapies (for example, sodium restriction and diuretics for cardiomyopathy) may also alleviate signs and symptoms of end-organ dysfunction. ${ }^{12}$ The most effective treatment for AL amyloidosis consists of high-dose melphalan (HDM) followed by autologous peripheral blood stem cell transplantation (SCT). Alternative therapies to HDM and SCT include cyclical low-dose oral melphalan with prednisone, continuous oral melphalan alone, and pulsed high-dose dexamethasone. ${ }^{2,12}$ Newer therapies include the use of immunomodulatory drugs (thalidomide and lenalidomide) and a proteasome inhibitor (bortezomib); these agents can be used with or without dexamethasone. ${ }^{2}$

Our patient refused systemic treatment regardless of diagnosis; therefore, the invasive bone marrow biopsy method of definitive diagnosis for systemic AL amyloid- osis was not pursued. However, given her normal FLC assay, SPEP, and IFE and lack of symptoms suggesting the involvement of other organ systems, the patient's AL amyloidosis is probably isolated to her right radial nerve. Without an underlying plasma cell dyscrasia, complete local excision is curative. Imaging characteristics of intraneural amyloid have not been well described. In this case, however, MRN showed a focal lesion versus a more diffuse process. In similar patients with nontraumatic mononeuropathy and imaging revealing a focal lesion in the corresponding nerve, intraneural amyloidoma should be included in the differential diagnosis. The timing of an operation should be based on clinical features and disease course.

\section{Conclusions}

While it is the most common form of amyloidosis, AL amyloidosis represents a rare disease, and isolated peripheral nerve disease is even rarer. Peripheral nerve involvement in systemic amyloidosis is usually characterized by symmetric polyneuropathy and can even be the first symptom of the disease. A review of the literature has shown peripheral nerve amyloidosis to fall within the context of a systemic plasma cell dyscrasia. However, it is important to recognize unique cases of isolated peripheral nerve $\mathrm{AL}$ amyloidosis with an intraneural mass detectable on MRN, as these can be managed with curative resection. At the same time, upon the discovery of peripheral neuropathy attributable to amyloidosis, clinical suspicion and investigation of systemic involvement is warranted because of the progressive nature of the disease toward multisystem organ failure.

\section{References}

1. Andrei IA, Kuntzer T, Lobrinus JA, Jaccard A, Zufferey P: Neuroarthropathy of the foot revealing primary systemic amyloidosis: case report and literature review. Clin Rheumatol [epub ahead of print], 2014

2. Baker KR, Rice L: The amyloidoses: clinical features, diagnosis and treatment. Methodist DeBakey Cardiovasc J 8:3-7, 2012

3. Berghoff M, Kathpal M, Khan F, Skinner M, Falk R, Freeman R: Endothelial dysfunction precedes C-fiber abnormalities in primary (AL) amyloidosis. Ann Neurol 53:725-730, 2003

4. Conaghan P, Chung D, Vaughan R: Recurrent laryngeal nerve palsy associated with mediastinal amyloidosis. Thorax 55:436-437, 2000

5. Dispenzieri A, Kyle RA, Lacy MQ, Therneau TM, Larson DR, Plevak MF, et al: Superior survival in primary systemic amyloidosis patients undergoing peripheral blood stem cell transplantation: a case-control study. Blood 103:3960-3963, 2004

6. Dispenzieri A, Lacy MQ, Kyle RA, Therneau TM, Larson DR, Rajkumar SV, et al: Eligibility for hematopoietic stem-cell transplantation for primary systemic amyloidosis is a favorable prognostic factor for survival. J Clin Oncol 19:3350-3356, 2001

7. Garces-Sanchez M, Dyck PJ, Kyle RA, Zeldenrust S, Wu Y, Ladha SS, et al: Antibodies to myelin-associated glycoprotein (anti-Mag) in IgM amyloidosis may influence expression of neuropathy in rare patients. Muscle Nerve 37:490-495, 2008 
8. Katoh N, Matsuda M, Yoshida T, Yazaki M, Morita H, Sakashita K, et al: Primary AL amyloid polyneuropathy successfully treated with high-dose melphalan followed by autologous stem cell transplantation. Muscle Nerve 41:138 143,2010

9. Katzmann JA, Abraham RS, Dispenzieri A, Lust JA, Kyle RA: Diagnostic performance of quantitative kappa and lambda free light chain assays in clinical practice. Clin Chem 51:878-881, 2005

10. Luigetti M, Papacci M, Bartoletti S, Marcaccio A, Romano A, Sabatelli M: AL amyloid neuropathy mimicking a chronic inflammatory demyelinating polyneuropathy. Amyloid 19:53-55, 2012

11. Matsuda M, Gono T, Morita H, Katoh N, Kodaira M, Ikeda $\mathrm{S}$ : Peripheral nerve involvement in primary systemic AL amyloidosis: a clinical and electrophysiological study. Eur J Neurol 18:604-610, 2011

12. Sanchorawala V: Light-chain (AL) amyloidosis: diagnosis and treatment. Clin J Am Soc Nephrol 1:1331-1341, 2006

13. Sarkar C, Chand Sharma M, Nayak A, Mercy Ralte A, Gupta V, Singh S, et al: Primary AL (kappa-light chain) amyloidosis manifesting as peripheral neuropathy in a young male without increase in serum and urine immunoglobulin load: a diagnostic challenge. Clin Neuropathol 24:118-125, 2005

14. Tracy JA, Dyck PJ, Dyck PJ: Primary amyloidosis presenting as upper limb multiple mononeuropathies. Muscle Nerve 41:710-715, 2010
15. Vucic S, Chong PS, Cros D: Atypical presentations of primary amyloid neuropathy. Muscle Nerve 28:696-702, 2003

16. Zanusso GL, Moretto G, Bonetti B, Monaco S, Rizzuto N: Complement neoantigen and vitronectin are components of plaques in amyloid AL neuropathy. Ital J Neurol Sci 13:493-499, 1992

\section{Disclosures}

The authors report no conflict of interest concerning the materials or methods used in this study or the findings specified in this paper.

\section{Author Contributions}

Conception and design: Cheng, Eskandari, Varma. Acquisition of data: Varma. Analysis and interpretation of data: Cheng, Welsh. Drafting the article: Cheng, Eskandari, Welsh. Critically revising the article: all authors. Reviewed submitted version of manuscript: all authors. Approved the final version of the manuscript on behalf of all authors: Cheng. Administrative/technical/material support: Welsh. Study supervision: Varma.

\section{Correspondence}

Ron Ron Cheng, Department of Neurosurgery, Medical University of South Carolina, 169 Ashley Ave., CSB 301, Charleston, SC 29425. email: cheng.ronron@gmail.com. 